\title{
Supporting Information Interaction between Monomeric Vanadium Oxide Clusters Supported on Titania and Its Influence on Their Reactivity
}

Henrik H. Kristoffersen and Horia Metiu*

Department of Chemistry and Biochemistry, University of California, Santa Barbara, California 93106-9510, United States

*E-mail: metiu@chem.ucsb.edu 


\section{S1 Bader charge}

The structures presented in the main manuscript have been subject to Bader analysis to obtain the total charge $\left(n_{t o t}\right)$ in the Bader volume of each atom and the difference between the spin-up and the spin-down electron density in the Bader volume of each atom $\left(n_{+}-n_{-}\right)$. The values for the $\mathrm{V}$ atoms are collected in Table S1.

Table S1: Bader charge $\left(n_{t o t}\right)$, Bader spin density difference $\left(n_{+}-n_{-}\right)$and the derived formal charge for the $\mathrm{V}$ atoms in this study.

\begin{tabular}{|l|c|c|c|c|c|c|}
\hline $\begin{array}{l}\text { System } \\
\left(\mathrm{V}(\alpha) \mathrm{O}_{x}, \mathrm{~V}(\beta) \mathrm{O}_{y}\right)\end{array}$ & $n_{\text {tot }}$ & $n_{+}-n_{-}$ & $\begin{array}{c}\text { formal } \\
\text { charge }\end{array}$ & $n_{\text {tot }}$ & $n_{+}-n_{-}$ & $\begin{array}{c}\text { formal } \\
\text { charge }\end{array}$ \\
\hline$\left(\mathrm{VO}_{2}, \mathrm{VO}_{2}\right)$ & $2.01 e$ & $1.02 e$ & $4+$ & $2.01 e$ & $-1.02 e$ & $4+$ \\
\hline$\left(\mathrm{VO}_{3}, \mathrm{VO}_{3}\right)$ & $2.19 e$ & $0.11 e$ & $5+$ & $2.19 e$ & $-0.11 e$ & $5+$ \\
\hline$\left(\mathrm{VO}_{3}^{-}, \mathrm{VO}_{2}^{+}\right)$ & $2.18 e$ & $0 e$ & $5+$ & $2.16 e$ & $0 e$ & $5+$ \\
\hline$\left.\left(\mathrm{VO}_{3}^{-}, \mathrm{OV}_{2} \mathrm{O}_{2}\right)^{+}\right)$ & $2.18 e$ & $0 e$ & $5+$ & $2.15 e$ & $0 e$ & $5+$ \\
\hline$\left(\mathrm{VO}_{2}, \mathrm{Ti}^{4+}\right)$ & $2.01 e$ & $1.02 e$ & $4+$ & & & \\
\hline$\left(\mathrm{VO}_{2}^{+}, \mathrm{Ti}^{3+}\right)^{a}$ & $2.14 e$ & $0 e$ & $5+$ & & & \\
\hline$\left(\mathrm{VO}_{2} \mathrm{H}_{2} \mathrm{VO}_{2}\right)$ & $1.84 e$ & $1.90 e$ & $3+$ & $2.01 e$ & $-1.02 e$ & $4+$ \\
\hline$\left(\mathrm{VO}_{3}, \mathrm{VO}_{3} \mathrm{H}\right)$ & $2.19 e$ & $-0.11 e$ & $5+$ & $2.19 e$ & $0 e$ & $5+$ \\
\hline$\left(\mathrm{VO}_{3} \mathrm{H}_{2} \mathrm{VO}_{2}\right)$ & $2.18 e$ & $0 e$ & $5+$ & $2.01 e$ & $1.02 e$ & $4+$ \\
\hline$\left(\mathrm{VO}_{3}, \mathrm{VO}_{3} \mathrm{H}\right)$ & $2.19 e$ & $-0.11 e$ & $5+$ & $2.19 e$ & $0 e$ & $5+$ \\
\hline
\end{tabular}

${ }^{a}$ The surface $5 c-\mathrm{Ti}^{3+}$ found in this system has $n_{\text {tot }}=2.05 e$ and $n_{+}-n_{-}=$ $0.84 e . \mathrm{Ti}^{4+}$ in the clean $\mathrm{TiO}_{2}(110)$ slab have $2.29 e \leq n_{\text {tot }} \leq 2.34 e$ and $n_{+}-n_{-}=0 e$. 


\section{S2 Vibrations}

We have calculated the vibrational spectrum of the $\left(\mathrm{VO}_{2}, \mathrm{VO}_{2}\right),\left(\mathrm{VO}_{3}, \mathrm{VO}_{3}\right)$, $\left(\mathrm{VO}_{3}^{-}, \mathrm{VO}_{2}^{+}\right)$and $\left(\mathrm{VO}_{3}^{-}, \mathrm{OV}\left(\mathrm{O}_{2}\right)^{+}\right)$systems (Table $\mathrm{S} 2$. The Hessian matrix is obtained by a finite difference approximation and only the vanadium atoms and the oxygen atoms bounded to the vanadium atoms are included. 
Table S2: Calculated vibrational frequencies $\left(\mathrm{cm}^{-1}\right)$.

\begin{tabular}{|c|c|c|c|}
\hline$\left(\mathrm{VO}_{2}, \mathrm{VO}_{2}\right)$ & $\left(\mathrm{VO}_{3}, \mathrm{VO}_{3}\right)$ & $\left(\mathrm{VO}_{3}^{-}, \mathrm{VO}_{2}^{+}\right)$ & $\left(\mathrm{VO}_{3}^{-}, \mathrm{OV}\left(\mathrm{O}_{2}\right)^{+}\right)$ \\
\hline 755.3 & $1043.9(\mathrm{~V}=\mathrm{O})$ & $1048.0(\mathrm{~V}=\mathrm{O})$ & $1057.4(\mathrm{~V}=\mathrm{O})$ \\
\hline 754.5 & $1041.5(\mathrm{~V}=\mathrm{O})$ & $1041.8(\mathrm{~V}=\mathrm{O})$ & $1042.6(\mathrm{~V}=\mathrm{O})$ \\
\hline 685.5 & 667.4 & 763.5 & $888.8(\mathrm{O}-\mathrm{O})$ \\
\hline 681.2 & 664.5 & 707.8 & 708.4 \\
\hline 555.1 & 566.5 & 675.4 & 701.0 \\
\hline 554.2 & 561.4 & 602.7 & 604.1 \\
\hline 528.7 & 499.7 & 581.9 & 597.5 \\
\hline 526.5 & 498.6 & 581.1 & 581.5 \\
\hline 470.6 & 482.2 & 546.6 & 547.8 \\
\hline 470.0 & 476.8 & 516.6 & 545.0 \\
\hline 467.9 & 474.2 & 498.2 & 516.9 \\
\hline 466.3 & 473.7 & 487.9 & 504.3 \\
\hline 441.0 & 441.0 & 487.4 & 498.2 \\
\hline 429.6 & 440.4 & 436.6 & 487.3 \\
\hline 393.2 & 439.9 & 425.8 & 452.9 \\
\hline 390.8 & 439.2 & 396.9 & 437.5 \\
\hline 382.4 & 409.8 & 377.6 & 429.5 \\
\hline 380.6 & 407.2 & 373.1 & 389.6 \\
\hline 346.3 & 396.9 & 347.4 & 377.9 \\
\hline 346.1 & 393.7 & 339.1 & 375.9 \\
\hline 340.5 & 327.7 & 310.3 & 347.7 \\
\hline 339.5 & 327.4 & 261.9 & 339.3 \\
\hline 301.0 & 273.7 & 252.2 & 317.7 \\
\hline 300.4 & 271.2 & 249.5 & 317.0 \\
\hline 231.7 & 242.2 & 242.1 & 248.0 \\
\hline 231.5 & 241.7 & 222.5 & 244.1 \\
\hline 204.6 & 240.0 & 202.3 & 242.3 \\
\hline 204.3 & 239.4 & 198.2 & 230.1 \\
\hline 182.4 & 197.5 & 188.4 & 222.5 \\
\hline \multirow[t]{7}{*}{180.9} & 197.3 & 145.5 & 197.7 \\
\hline & 192.2 & 144.7 & 192.9 \\
\hline & 191.7 & 132.2 & 168.9 \\
\hline & 139.8 & 107.8 & 141.4 \\
\hline & 136.8 & & 132.0 \\
\hline & 124.8 & & 124.2 \\
\hline & 123.9 & & 118.1 \\
\hline
\end{tabular}




\section{S3 Selected thermodynamic data}

Table S3: Thermodynamic data used to compare the relative abundance of $\left(\mathrm{VO}_{3}^{-}, \mathrm{VO}_{2}^{+}\right)$and $\left(\mathrm{VO}_{3}^{-}, \mathrm{OV}\left(\mathrm{O}_{2}\right)^{+}\right)$

\begin{tabular}{|l|c|c|c|c|}
\hline & $\mathrm{O}_{2}(\mathrm{~g})(1 \mathrm{~atm})^{a}$ & $\left(\mathrm{VO}_{3}^{-}, \mathrm{VO}_{2}^{+}\right)$ & $\left(\mathrm{VO}_{3}^{-}, \mathrm{OV}\left(\mathrm{O}_{2}\right)^{+}\right)$ & $\begin{array}{c}\left(\mathrm{VO}_{3}^{-}, \mathrm{VO}_{2}^{+}\right) \\
+\frac{1}{2} \mathrm{O}_{2}(\mathrm{~g}) \rightarrow \\
\left(\mathrm{VO}_{3}^{-}, \mathrm{OV}^{\circ}\left(\mathrm{O}_{2}\right)^{+}\right)\end{array}$ \\
\hline$E_{f}$ & $0.00 \mathrm{eV}$ & $-14.90 \mathrm{eV}$ & $-15.12 \mathrm{eV}$ & $-0.22 \mathrm{eV}$ \\
$\mathrm{ZPE}$ & $0.10 \mathrm{eV}$ & $0.86 \mathrm{eV}$ & $0.95 \mathrm{eV}$ & $0.04 \mathrm{eV}$ \\
$\Delta G^{0}(0 \mathrm{~K})$ & $0.10 \mathrm{eV}$ & $-14.04 \mathrm{eV}$ & $-14.17 \mathrm{eV}$ & $-0.19 \mathrm{eV}$ \\
\hline$\Delta H^{0}(100 \mathrm{~K})$ & $0.13 \mathrm{eV}$ & $-14.01 \mathrm{eV}$ & $-14.15 \mathrm{eV}$ & $-0.20 \mathrm{eV}$ \\
$-T \Delta S^{0}(100 \mathrm{~K})$ & $-0.18 \mathrm{eV}$ & $-0.03 \mathrm{eV}$ & $-0.03 \mathrm{eV}$ & $0.09 \mathrm{eV}$ \\
$\Delta G^{0}(100 \mathrm{~K})$ & $-0.05 \mathrm{eV}$ & $-14.04 \mathrm{eV}$ & $-14.18 \mathrm{eV}$ & $-0.11 \mathrm{eV}$ \\
\hline$\Delta H^{0}(200 \mathrm{~K})$ & $0.16 \mathrm{eV}$ & $-13.90 \mathrm{eV}$ & $-14.03 \mathrm{eV}$ & $-0.21 \mathrm{eV}$ \\
$-T \Delta S^{0}(200 \mathrm{~K})$ & $-0.40 \mathrm{eV}$ & $-0.22 \mathrm{eV}$ & $-0.03 \mathrm{eV}$ & $0.18 \mathrm{eV}$ \\
$\Delta G^{0}(200 \mathrm{~K})$ & $-0.24 \mathrm{eV}$ & $-14.11 \mathrm{eV}$ & $-14.26 \mathrm{eV}$ & $-0.02 \mathrm{eV}$ \\
\hline$\Delta H^{0}(300 \mathrm{~K})$ & $0.19 \mathrm{eV}$ & $-13.72 \mathrm{eV}$ & $-13.83 \mathrm{eV}$ & $-0.21 \mathrm{eV}$ \\
$-T \Delta S^{0}(300 \mathrm{~K})$ & $-0.64 \mathrm{eV}$ & $-0.54 \mathrm{eV}$ & $-0.58 \mathrm{eV}$ & $0.28 \mathrm{eV}$ \\
$\Delta G^{0}(300 \mathrm{~K})$ & $-0.45 \mathrm{eV}$ & $-14.26 \mathrm{eV}$ & $-14.41 \mathrm{eV}$ & $0.07 \mathrm{eV}$ \\
\hline$\Delta H^{0}(400 \mathrm{~K})$ & $0.22 \mathrm{eV}$ & $-13.50 \mathrm{eV}$ & $-13.59 \mathrm{eV}$ & $-0.20 \mathrm{eV}$ \\
$-T \Delta S^{0}(400 \mathrm{~K})$ & $-0.89 \mathrm{eV}$ & $-0.97 \mathrm{eV}$ & $-1.05 \mathrm{eV}$ & $0.37 \mathrm{eV}$ \\
$\Delta G^{0}(400 \mathrm{~K})$ & $-0.67 \mathrm{eV}$ & $-14.47 \mathrm{eV}$ & $-14.64 \mathrm{eV}$ & $0.16 \mathrm{eV}$ \\
\hline
\end{tabular}

${ }^{a}$ http://kinetics.nist.gov/janaf/

${ }^{b}$ Zero point energies (ZPE) calculated from the vibrational frequencies. 


\section{S4 Effect of $\mathrm{U}$ value applied to $\mathrm{V}$ d-states}

The mutual stabilization of the pair $\left(\mathrm{VO}_{2}, \mathrm{VO}_{3}\right)$ through charge transfer is the main result of this paper. We have therefore calculated the formation energies of the clusters in Figure 2 with different $\mathrm{U}$ values applied to the $\mathrm{V}$ d-states (Table $\mathrm{S} 4$ ). $\mathrm{U}$ value of $2.5 \mathrm{eV}$ or $4.5 \mathrm{eV}$ instead of $3.5 \mathrm{eV}$, change the formation energies, but the mutual stability is unchanged for $\left(\mathrm{VO}_{3}, \mathrm{VO}_{3}\right),\left(\mathrm{VO}_{3}^{-}, \mathrm{VO}_{2}^{+}\right)$, and $\left(\mathrm{VO}_{3}^{-}, \mathrm{OV}\left(\mathrm{O}_{2}\right)^{+}\right)$, where the $\mathrm{V}$ atoms have formal charges of $5+$. The $\mathrm{V}$ atoms in $\left(\mathrm{VO}_{2}, \mathrm{VO}_{2}\right)$ have $4+$ formal charges and the formation energy is less affected by changing the $\mathrm{U}$ value. However, $\left(\mathrm{VO}_{2}, \mathrm{VO}_{2}\right)$ remains significantly less stable than the other clusters.

Table S4: Formation energies $\left(E_{f}\right)$ for the vanadium oxide clusters calculated according to eq 1 in the paper, but with different $\mathrm{U}$ values applied to the $\mathrm{V}$ d-states. The $\mathrm{U}$ value applied to Ti d-states is $3.5 \mathrm{eV}$.

\begin{tabular}{|c|c|c|c|c|}
\hline $\mathrm{U}$ on V d-states & $\left(\mathrm{VO}_{2}, \mathrm{VO}_{2}\right)$ & $\left(\mathrm{VO}_{3}, \mathrm{VO}_{3}\right)$ & $\left(\mathrm{VO}_{3}^{-}, \mathrm{VO}_{2}^{+}\right)$ & $\left(\mathrm{VO}_{3}^{-}, \mathrm{OV}\left(\mathrm{O}_{2}\right)^{+}\right)$ \\
\hline $2.5 \mathrm{eV}$ & $-13.21 \mathrm{eV}$ & $-14.69 \mathrm{eV}$ & $-15.51 \mathrm{eV}$ & $-15.72 \mathrm{eV}$ \\
\hline $3.5 \mathrm{eV}$ & $-13.05 \mathrm{eV}$ & $-14.12 \mathrm{eV}$ & $-14.90 \mathrm{eV}$ & $-15.12 \mathrm{eV}$ \\
\hline $4.5 \mathrm{eV}$ & $-12.47 \mathrm{eV}$ & $-13.10 \mathrm{eV}$ & $-13.85 \mathrm{eV}$ & $-14.09 \mathrm{eV}$ \\
\hline
\end{tabular}




\section{S5 Additional cluster structures}

In this section we compare structures presented in the main paper (Image (a) in

Figure S1 to S5) to other cluster structures containing the same total number of atoms.

(a)

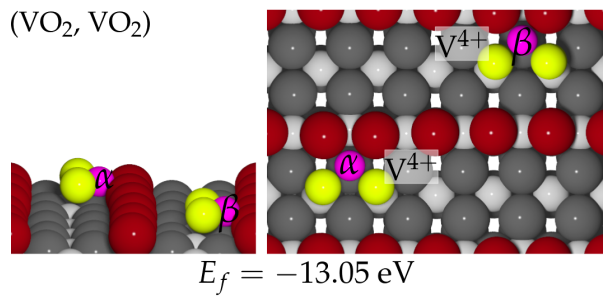

(b)

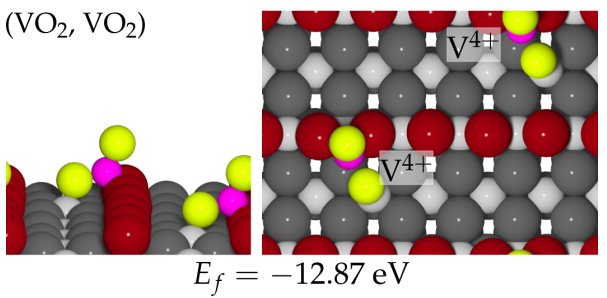

Figure S1: (a) Figure 2a in the main paper showing the lowest energy $\left(\mathrm{VO}_{2}\right.$, $\left.\mathrm{VO}_{2}\right)$ configuration. (b) A less stable $\left(\mathrm{VO}_{2}, \mathrm{VO}_{2}\right)$ configuration.

(a)

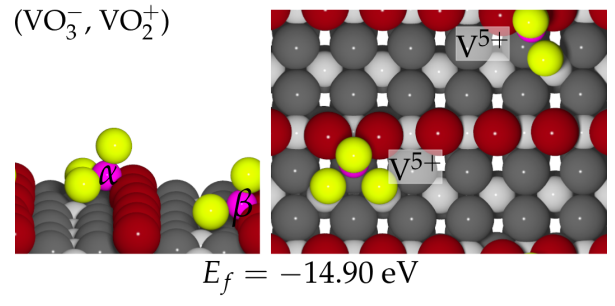

(b)

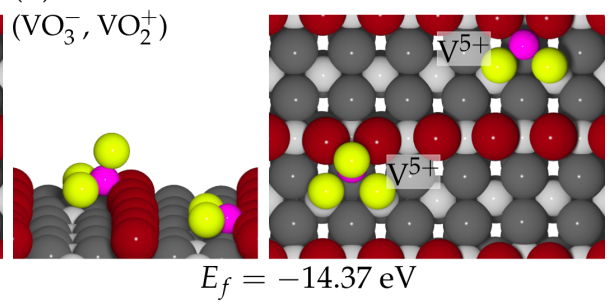

Figure $\mathrm{S} 2$ : (a) Figure 2e in the main paper showing the lowest energy $\left(\mathrm{VO}_{3}^{-}\right.$, $\mathrm{VO}_{2}^{+}$) configuration. (b) A less stable $\left(\mathrm{VO}_{3}^{-}, \mathrm{VO}_{2}^{+}\right)$configuration. 
(a)
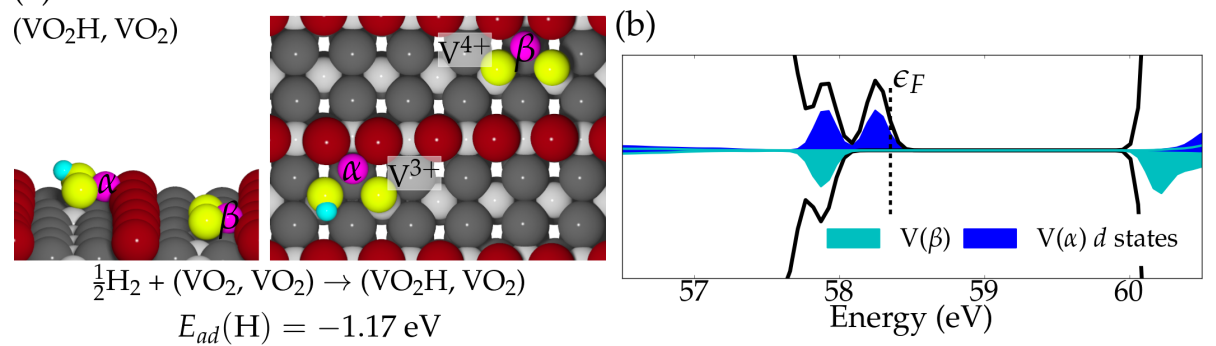

(c)
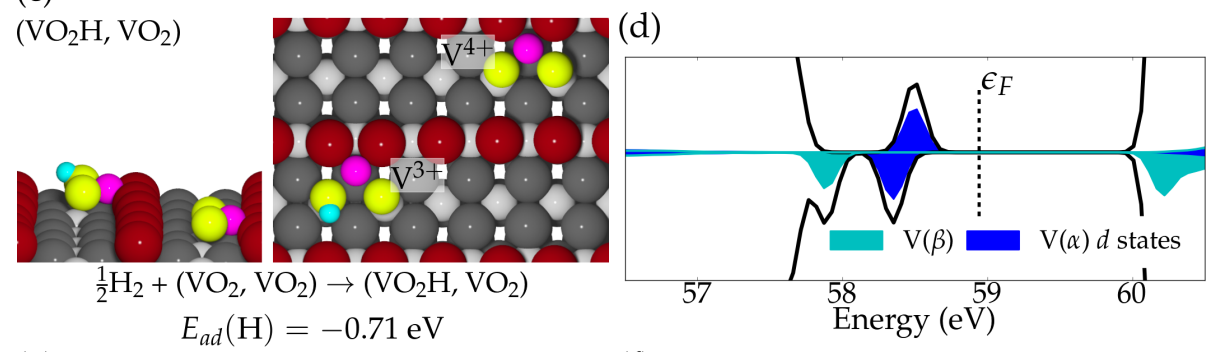

(e)

(f)

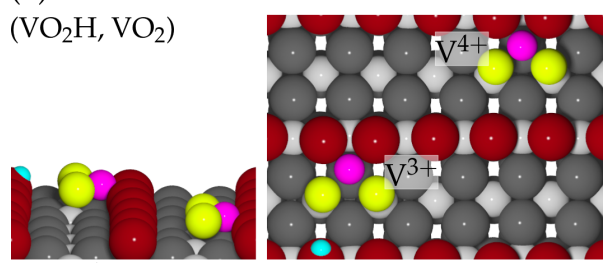

$$
\begin{gathered}
\frac{1}{2} \mathrm{H}_{2}+\left(\mathrm{VO}_{2}, \mathrm{VO}_{2}\right) \rightarrow\left(\mathrm{VO}_{2} \mathrm{H}, \mathrm{VO}_{2}\right) \\
E_{a d}(\mathrm{H})=-0.96 \mathrm{eV}
\end{gathered}
$$

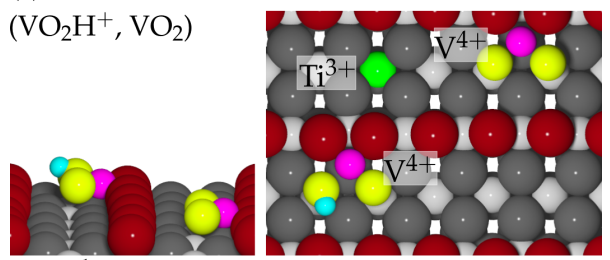

$$
\begin{gathered}
\frac{1}{2} \mathrm{H}_{2}+\left(\mathrm{VO}_{2}, \mathrm{VO}_{2}\right) \rightarrow\left(\mathrm{VO}_{2} \mathrm{H}^{+}, \mathrm{VO}_{2}\right) \\
E_{a d}(\mathrm{H})=-0.31 \mathrm{eV}
\end{gathered}
$$

Figure S3: (a) and (b) are Figure $4 \mathrm{a}$ and $4 \mathrm{~b}$ in the main paper showing the configuration and PDOS for the lowest energy $\left(\mathrm{VO}_{2} \mathrm{H}, \mathrm{VO}_{2}\right)$ configuration obtained by $\frac{1}{2} \mathrm{H}_{2}$ adsorption on $\left(\mathrm{VO}_{2}, \mathrm{VO}_{2}\right)$. (c) and (d) shows the same atomic configuration as (a) and (b), but with the two electron on $\mathrm{V}^{3+}(\mathrm{V}(\alpha))$ having opposite spin instead of aligned spin. (e) and (f) show less stable configurations with the same number of atoms. In (f) one electron is moved from $\mathrm{VO}_{2} \mathrm{H}^{+}$to a $5 c$-Ti in the surface. 
(a)

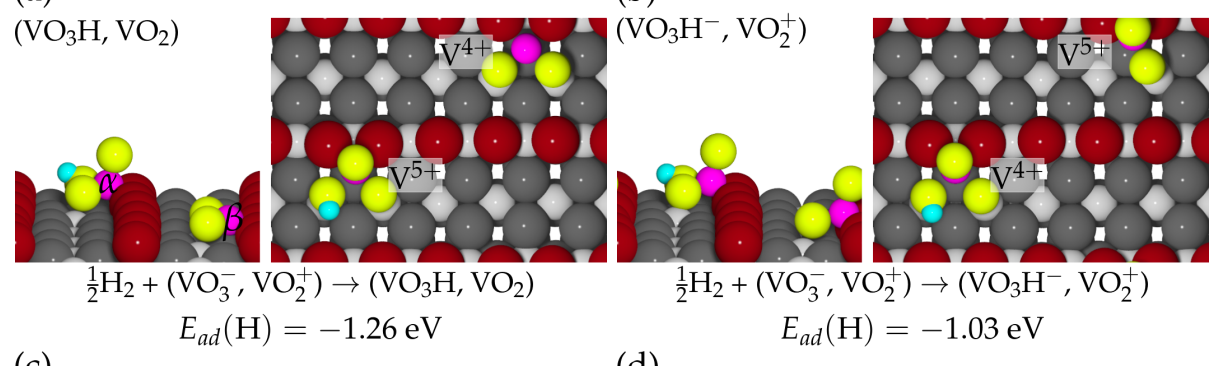

(c)

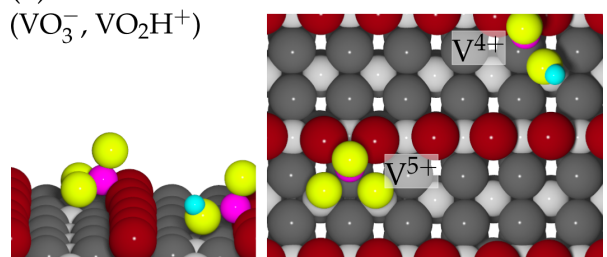

$\frac{1}{2} \mathrm{H}_{2}+\left(\mathrm{VO}_{3}^{-}, \mathrm{VO}_{2}^{+}\right) \rightarrow\left(\mathrm{VO}_{3}^{-}, \mathrm{VO}_{2} \mathrm{H}^{+}\right)$

$E_{a d}(\mathrm{H})=-1.08 \mathrm{eV}$

(e)

$\left(\mathrm{VO}_{3} \mathrm{H}, \mathrm{VO}_{2}\right)$

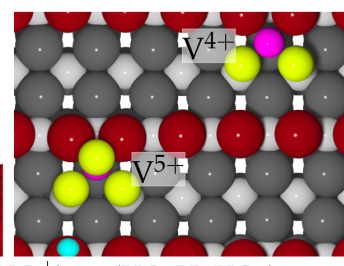

$\frac{1}{2} \mathrm{H}_{2}+\left(\mathrm{VO}_{3}^{-}, \mathrm{VO}_{2}^{+}\right) \rightarrow\left(\mathrm{VO}_{3} \mathrm{H}, \mathrm{VO}_{2}\right)$

$$
E_{a d}(\mathrm{H})=-1.17 \mathrm{eV}
$$

(g)

$\left(\mathrm{VO}_{3} \mathrm{H}, \mathrm{VO}_{2}\right)$

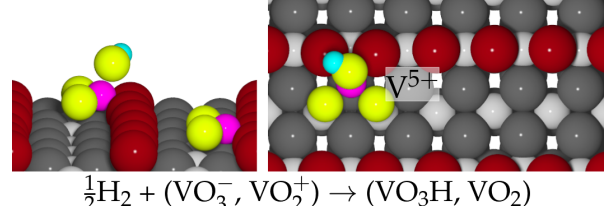

$$
E_{a d}(\mathrm{H})=-1.04 \mathrm{eV}
$$

(f)

$\left(\mathrm{VO}_{3}^{-}, \mathrm{VO}_{2} \mathrm{H}^{+}\right)$

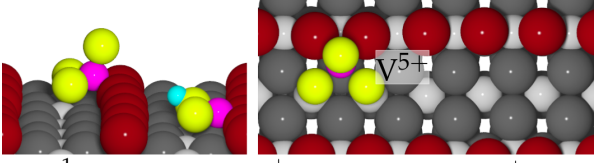

$\frac{1}{2} \mathrm{H}_{2}+\left(\mathrm{VO}_{3}^{-}, \mathrm{VO}_{2}^{+}\right) \rightarrow\left(\mathrm{VO}_{3}^{-}, \mathrm{VO}_{2} \mathrm{H}^{+}\right)$

$E_{\text {ad }}(\mathrm{H})=-1.11 \mathrm{eV}$

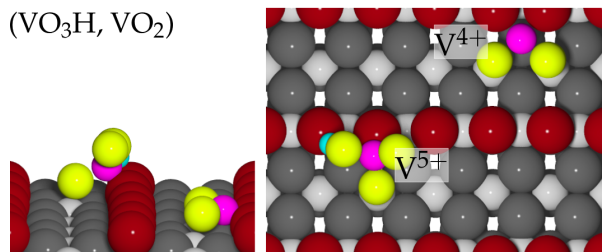

$\frac{1}{2} \mathrm{H}_{2}+\left(\mathrm{VO}_{3}^{-}, \mathrm{VO}_{2}^{+}\right) \rightarrow\left(\mathrm{VO}_{3} \mathrm{H}, \mathrm{VO}_{2}\right)$

$E_{a d}(\mathrm{H})=-1.05 \mathrm{eV}$

Figure S4: (a) Figure 4e in the main paper showing the lowest energy $\left(\mathrm{VO}_{3} \mathrm{H}\right.$, $\mathrm{VO}_{2}$ ) configuration obtained by $\frac{1}{2} \mathrm{H}_{2}$ adsorption on $\left(\mathrm{VO}_{3}^{-}, \mathrm{VO}_{2}^{+}\right)$. (b), (c), (d), (e), (f) and (g) show less stable configurations with the same number of atoms. 
(a)

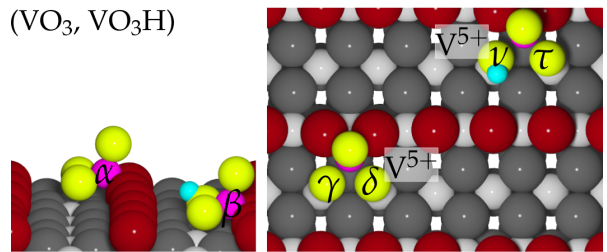

$\frac{1}{2} \mathrm{H}_{2}+\left(\mathrm{VO}_{3}^{-}, \mathrm{OV}\left(\mathrm{O}_{2}\right)^{+}\right) \rightarrow\left(\mathrm{VO}_{3}, \mathrm{VO}_{3} \mathrm{H}\right)$

$E_{\text {ad }}(\mathrm{H})=-1.56 \mathrm{eV}$

(c)

$\left(\mathrm{VO}_{3}^{-}, \mathrm{OV}\left(\mathrm{O}_{2} \mathrm{H}\right)^{+}\right)$
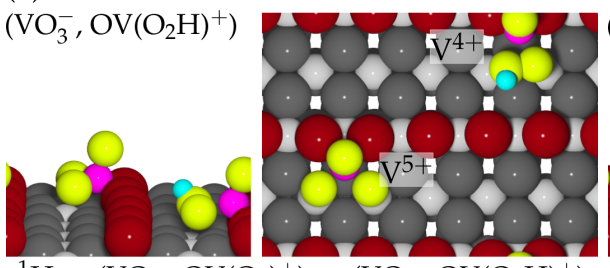

$\frac{1}{2} \mathrm{H}_{2}+\left(\mathrm{VO}_{3}^{-}, \mathrm{OV}\left(\mathrm{O}_{2}\right)^{+}\right) \rightarrow\left(\mathrm{VO}_{3}^{-}, \mathrm{OV}\left(\mathrm{O}_{2} \mathrm{H}\right)^{+}\right)$

$E_{a d}(\mathrm{H})=-0.26 \mathrm{eV}$ (b)

$\left(\mathrm{VO}_{3}, \mathrm{VO}_{3} \mathrm{H}\right)$
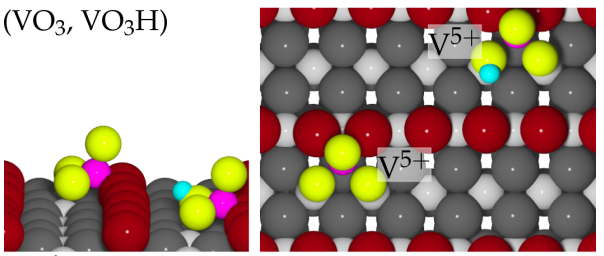

$\frac{1}{2} \mathrm{H}_{2}+\left(\mathrm{VO}_{3}^{-}, \mathrm{OV}\left(\mathrm{O}_{2}\right)^{+}\right) \rightarrow\left(\mathrm{VO}_{3}, \mathrm{VO}_{3} \mathrm{H}\right)$

(d)

$E_{a d}(\mathrm{H})=-1.45 \mathrm{eV}$

$\left(\mathrm{VO}_{3} \mathrm{H}, \mathrm{OV}\left(\mathrm{O}_{2}\right)^{+}\right)$

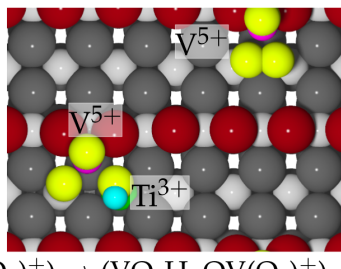

$\frac{1}{2} \mathrm{H}_{2}+\left(\mathrm{VO}_{3}^{-}, \mathrm{OV}\left(\mathrm{O}_{2}\right)^{+}\right) \rightarrow\left(\mathrm{VO}_{3} \mathrm{H}, \mathrm{OV}\left(\mathrm{O}_{2}\right)^{+}\right)$

$$
E_{\text {ad }}(\mathrm{H})=-0.40 \mathrm{eV}
$$

Figure S5: (a) Figure $4 \mathrm{~g}$ in the main paper showing the stable $\left(\mathrm{VO}_{3}, \mathrm{VO}_{3} \mathrm{H}\right)$ configuration obtained by $\frac{1}{2} \mathrm{H}_{2}$ adsorption on $\left(\mathrm{VO}_{3}^{-}, \mathrm{OV}\left(\mathrm{O}_{2}\right)^{+}\right)$. (b), (c) and (d) show less stable configurations with the same number of atoms. The subfigures (a) and (b) differ by the location of the low lying empty orbital, which is located on the $\mathrm{VO}_{3}$ cluster in (a) and in the support in (b). 